\title{
Das Reich der Wolke
}

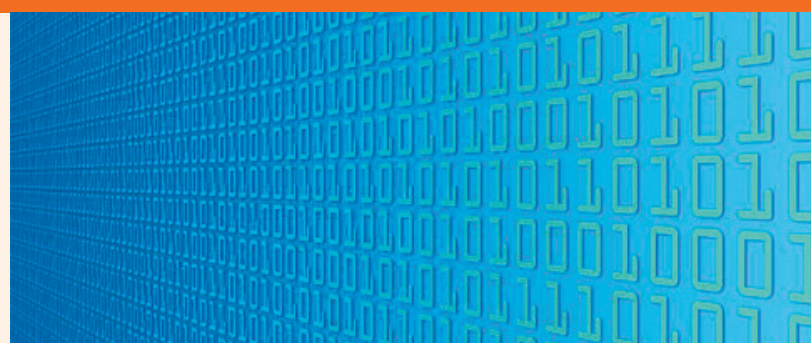

Erhard Taverna

1 Vgl.: Die Gazette, das politische Kulturmagazin. München: 2009;23. www.gazette.de

2 Neffe J. Kulturwandel. Es war einmal. www.zeit. de/2009/18/L-Buch

3 Flusser V. Die Schrift: Hat Schreiben Zukunft? Göttingen: Edition Immatrix; 1992.

Das neuste Google-Angebot handelt für einmal nicht von einer neuen Technik. Cloud Computing ist vorerst noch der Entwurf eines Netzwerks verschiedener Dienste im Internet. Die Wolke entlastet uns von Dateien und Programmen, denn sie werden in virtuelle Ablagen ausgelagert. Ein Sparmodell, dynamisch und skalierbar, anwenderfreundlich und kostensparend, mit unbeschränktem Zugriff. Mit «Book Search» digitalisiert die gleiche Suchmaschine Bücher und Bibliotheken auf der ganzen Welt, immer im Namen der globalen Wissensverbreitung. Diese «Bibliothek in der Wolke» lädt kein vollständiges E-Book auf ein Lesegerät. Geboten wird nur ein Zugang, die Bücher bleiben auf dem Server. Google ist zum Motor geworden, der das ganze Internet vorantreibt. Dazu gehören neben vielen bekannten Diensten ein eigenes Handy (G1), ein eigenes Betriebssystem (Android), Google Mail, der Browser Chrome und die Buchsuche für Millionen eingescannter Titel. Inzwischen werden auch in Europa im Portal «Europeana» Bibliotheken und urheberrechtlich geschütztes Material kostenlos dem Kunden überlassen [1].

Dass die Ära des gedruckten Buches zu Ende gehe, wird schon seit Jahren vorausgesagt, von den einen freudig begrüsst, von anderen mit Sorge beobachtet. Für viele sind die erhältlichen E-Books als Buchersatzmaschinen bereits überholt. Sie schwärmen von Büchern, die auf allen möglichen Geräten lesbar werden, gleichzeitig Bilder anzeigen, Töne abspielen und diese über jede Menge Links mit weiteren Informationen und Kommentaren verknüpfen. Die Grenzen zwischen dem Buch und dem Rest der Medienwelt würden sich schliesslich so vollständig verlieren wie die zwischen Werbung und Unterhaltung [2]. Der neue Hypertext wird das altmodische, lineare Lesen verdrängen. Im äusseren Erscheinungsbild wird die Schrift weniger selbständig auftreten. Wie bei Vorträgen mit Beamer-Projektion oft sichtbar, ist sie nur noch ein Element unter anderen, mehr oder weniger integriert in ein komplexes, multimediales Zeichengebilde. Schnelligkeit, Zerstückelung und Abwechslung sind wichtiger als Kontinuität und Ruhe. Was für didaktische und enzyklopädische Zwecke geeignet erscheint, wird zur allgemeinen Darbietungsform. Wenn einmal elektronische, hochfunktionelle Speicher das Papier ersetzen, werden die «Leser» neben wenigen Restschriften vor allem Symbole übersetzen. Wird dann überhaupt noch weiter geschrieben? Und führt allein schon die Missachtung der Urheberrechte dazu, dass aus der denkbaren Verödung der Buchkultur der Nachwuchs an geistigem Eigentum verödet?

Utopien aufgeschlossene Kommunikationsforscher wie Vilém Flusser (1920-1991) beschäftigt seit langem die Zukunft des alphabetischen Denkens. Flusser identifiziert die Schrift mit dem Code der Aufklärung [3]. Wie das Alphabet die Macht des Bildes abgelöst habe, würden die digitalen Codes das Geschichtsbewusstsein der traditionellen Buchkultur auslöschen. Der Philosoph stellt sich dabei einen zukünftigen Leser vor, wie dieser aus praktisch unendlichen Querverbindungen verfügbarer Informationselemente die beabsichtigte Information herstellt. Der mit der Textund Bildinflation vernetzte User erlebe in seiner künstlichen Raum- und Zeitkapsel eine ständige Gegenwart. Anstelle eines kohärenten, lebenslänglich erweiterten Wissens tritt der punktuelle, erinnerungslose Zugriff. Das Vergessen gehört zum neuen System, das Leben wird zur Benutzeroberfläche. Die Welt ist alles, was bei Google der Fall ist.

Man kann das als Spekulationen eines Buchmenschen abtun, der sorgenvoll in eine Zukunft blickt, der er nicht mehr angehört. Doch Vorsicht ist angebracht. Das Tempo der profitgetriebenen Informationstechnologien überfordert Politik und Recht. Kollateralschäden sind deutliche Warnzeichen. Zum Beispiel missachtete Urheberrechte bei einem zurzeit dominanten amerikanischen Recht, ungeklärter Datenschutz oder das Vorgehen von Amazon beim Lesegerät «Kindle». Von Kunden legal erworbene E-Books wurden von ihren Geräten gelöscht, weil der Verlag diese illegal in Umlauf gebracht hatte. Die Besitzer wurden zwar entschädigt, zuvor aber nicht informiert. Auch Apple hat bei seinem iPhone-App-Store die Möglichkeit, installierte Anwendungen nachträglich zu löschen. Das Kundenbewusstsein für Missbrauchs- und Überwachungsmöglichkeiten ist völlig unterentwickelt. Zahlreiche Leserbriefe kritisieren den eidgenössischen Datenschützer, wenn er dem Internetdienst Google Street View Auflagen macht. Wer sich nichts zuschulden kommen lässt, hat angeblich keine Probleme. Dabei gibt es in einer digitalen Welt keine Privatsphäre. Alle sind jederzeit kontrollierbar, auch dort, wo neue Dienste virtuelle Datensafes anbieten.

Wer Informationen besitzt, hat die Macht und wird sie auch brauchen. Überwiegend kommerziell, doch das kann sich rasch ändern. Informationsinhalte können umgeschrieben, Bücher gelöscht und Links manipuliert werden. Altmodische Bücherleser sind vielleicht resistenter gegen die Sirenenklänge von Cyberworld, nützen wird es ihnen wohl nichts mehr. Gefordert sind mitdenkende Bürger und Bürgerinnen, die im Wettlauf gegen die Zeit ihre eigene Privatsphäre verteidigen und kritische Politiker, Juristen und Datenschützer bei ihren Bemühungen um eine öffentliche Kontrolle des Internets unterstützen. 